\title{
Downregulation of cold-inducible RNA-binding protein activates mitogen-activated protein kinases and impairs spermatogenic function in mouse testes
}

\author{
Zhi-Ping Xia ${ }^{1}$, Xin-Min Zheng ${ }^{1}$, Hang Zheng ${ }^{1}$, Xiao-Jun Liu ${ }^{1}$, Gui-Yong Liu ${ }^{2}$ and Xing-Huan Wang ${ }^{1}$ \\ Cold-inducible RNA-binding protein (CIRP) is an RNA-binding protein that is expressed in normal testes and downregulated after heat \\ stress caused by cryptorchidism, varicocele or environmental temperatures. The purpose of this study was to investigate the functions \\ of CIRP in the testes. We employed RNAi technique to knock down the expression of CIRP in the testes, and performed haematoxylin \\ and eosin staining to evaluate morphological changes following knockdown. Germ cell apoptosis was examined by terminal \\ deoxynucleotidal transferase-mediated dUTP nick end labelling (TUNEL) assay, and mitogen-activated protein kinase (MAPK) \\ signalling pathways were investigated by Western blotting to determine the possible mechanism of apoptosis. We found that using \\ siRNA is a feasible and reliable method for knocking down gene expression in the testes. Compared to controls, the mean seminiferous \\ tubule diameter (MSTD) and the thickness of the germ cell layers decreased following siRNA treatment, whereas the percentage of \\ apoptotic seminiferous tubules increased. The p44/p42, p38 and SAPK/JNK MAPK pathways were activated after downregulation of \\ CIRP. In conclusion, we discovered that downregulation of CIRP resulted in increased germ cell apoptosis, possibly via the activation of \\ the p44/p42, p38 and SAPK/JNK MAPK pathways.
}

Asian Journal of Andrology (2012) 14, 884-889; doi:10.1038/aja.2012.71; published online 24 September 2012

Keywords: cold-inducible RNA-binding protein (CIRP); mitogen-activated protein kinase (MAPK); siRNA in vivo; spermatogenesis; heat stress; male infertility

\section{INTRODUCTION}

RNA-binding proteins play important roles in spermatogenesis in the mammalian testes, and functional deficiencies of RNA-binding proteins may cause infertility. ${ }^{1-4}$ Cold-inducible RNA-binding protein (CIRP) is a highly conserved glycine-rich RNA-binding protein that contains an amino-terminal consensus sequence RNA-binding domain and a carboxy-terminal glycine-rich domain. ${ }^{5,6}$ CIRP was observed in response to stresses such as ultraviolet (UV) irradiation, hypoxia ${ }^{7}$ and moderately low temperatures. ${ }^{8}$ CIRP was first discovered in transcripts induced by DNA damage as a result of UV irradiation or UV mimetic agents, and the level of CIRP was found to be increased in a dosedependent manner in response to UV..$^{9,10}$ Overexpression of CIRP in colon carcinoma RKO cells was found to enhance translation in a dosedependent manner, and cells that expressed reduced levels of CIRP were shown to be more sensitive to UV radiation. ${ }^{11}$ CIRP has also been observed in response to hypoxia through a hypoxia inducible factor1-independent mechanism ${ }^{7}$ and is considered to be a stress-inducible protein that participates in the cellular response to oxidative stress. ${ }^{12}$ CIRP has been further reported to be induced by mild cold-shock ${ }^{13,14}$ and is involved in the response to several events that modulate transcription, translation, the cytoskeleton, the cell cycle and metabolic processes. ${ }^{15,16} \mathrm{CIRP}$ has more recently been identified as part of a new generation of proto-oncogenes. ${ }^{12}$
CIRP is constitutively expressed in the testes, ${ }^{17,18}$ but has not been well studied. Nishiyama et al. ${ }^{18}$ found that the expression of CIRP decreased in male germ cells at elevated temperatures, and Zhou et al. ${ }^{19}$ reported that overexpression of CIRP reduced the testicular damage induced by experimental cryptorchidism. It is well known that spermatogenesis is a temperature-dependent process and that spermatogenic function is impaired after heat stress in a complex pathological process involving hypoxia and oxidative stress. Hypoxia and high levels of free radicals and reactive oxygen species have been shown to result in cell cycle arrest and apoptosis ${ }^{20,21}$ and to contribute to male fertility problems. ${ }^{22-24}$ Other studies have indicated that CIRP reduces the damage caused by hypoxia and oxidative stress ${ }^{7,12}$ but it's downregulated after heat stress in the testes, ${ }^{18,19}$ this disorder may result in serious damage to spermatogenic function. The purpose of this study was to investigate the role of down-regulated CIRP in the testes to improve our understanding of the mechanism by which heat stress causes male infertility.

\section{MATERIALS AND METHODS}

Animals

$\mathrm{BALB} / \mathrm{c}$ mice (8 weeks old) were purchased from the Experimental Animal Center of Wuhan University (Wuhan, China) and were raised in the ABSL-3 laboratory of Wuhan University. All animal procedures

${ }^{1}$ Department of Urology, Zhongnan Hospital of Wuhan University, Wuhan 430071, China and 2Department of Urology, Qianjiang Central Hospital, Qianjiang 433100, China Correspondence: Dr XM Zheng (drzhengxinmin@sohu.com)

Received: 28 March 2012; Revised: 1 May 2012; Accepted: 5 June 2012; Published online: 24 September 2012 
used in this study conformed to NIH guidelines and were approved by the Ethics Committee of Wuhan University.

\section{Reagents}

Anti-CIRP antibodies for Western blotting and immunohistochemistry were obtained from the ProteinTech Group (Chicago, IL, USA); anti- $\beta$-Actin and anti-GAPDH antibodies were obtained from Santa Cruz Biotechnology (Santa Cruz, CA, USA). Anti-phospho-MAPK and anti-MAPK antibodies were obtained from Cell Signaling Technology (Danvers, MA, USA). The Lipofectamine 2000 reagent was obtained from Invitrogen (Grand Island, NY, USA).

\section{Immunohistochemical studies}

Immunohistochemistry was employed to determine the expression profile of CIRP in the testes. Testes tissues were fixed in formalin, embedded in paraffin and sectioned at $5 \mu \mathrm{m}$, and the immunohistochemical streptavidin-biotin complex method (SABC) was performed. Briefly, endogenous peroxidase activity was inhibited by $3 \%$ Hydrogen peroxide in distilled water for $10 \mathrm{~min}$. The sections were microwaved in antigen retrieval solution $\left(0.01 \mathrm{~mol} \mathrm{l}^{-1} \mathrm{pH} 6.0\right.$ citrate buffer) and were blocked with 5\% bovine serum albumin for $20 \mathrm{~min}$ The samples were incubated with the anti-CIRP antibody diluted $1: 100$ for $1 \mathrm{~h}$ at room temperature and were washed in phosphatebuffered saline (PBS) for $3 \times 2 \mathrm{~min}$. Biotinylated anti-rabbit IgG was added, and the samples were then washed in PBS for $3 \times 2 \mathrm{~min}$. Sections were incubated with an SABC complex (Boster, Wuhan, China) and were washed in PBS for $4 \times 5 \mathrm{~min}$. Peroxidase was detected with a DAB kit (Boster, Wuhan, China), and the samples were washed in tap water for $10 \mathrm{~min}$ and then dehydrated. The nuclei were stained with haematoxylin, and the sections were sealed with neutral balsam.

\section{Transient transfection of siRNA in the testes in vivo}

The CIRP-directed siRNA sequences (siRNA group) and nonsense siRNA sequences (negative control group, NC group) are shown in Table 1. The sequences were designed on the Dharmacon.com website (http://www.dharmacon.com/) and synthesized by Dharmacon (Chicago, IL, USA) with chemical modifications including 2'-Ome, phosphorylation and cholesterol modifications. Normal testes were taken as a control group. Approximately $10 \mu \mathrm{g}$ of the siRNA sequences was diluted in $20 \mu \mathrm{l} \mathrm{PBS}$ and incubated with $30 \mu \mathrm{l}$ of the Lipofectamine 2000 reagent at room temperature for $20 \mathrm{~min}$. Thirty microlitres of the complex was transfected into the seminiferous tubules using the microinjection method described by Ogawa et al..$^{25}$ The infiltration efficiency of the siRNA complex was evaluated by a trypan blue tracer $(0.04 \%)$ in frozen sections. Testes were harvested after $48 \mathrm{~h}$. All of the testes were divided into two parts, one for knockdown validation by western blotting, and the other for the following detections.

\section{Histopathological examination}

Testicular spermatogenesis was indicated by the mean seminiferous tubular diameter (MSTD) and the thickness of the germ cell layers.
Validated CIRP knockdown samples, siRNA-negative control samples and normal testes were prepared for histological examination. Testicular sections were fixed in formalin solution and stained with haematoxylin and eosin. The diameters and thickness of the germ cell layers of the seminiferous tubules were measured in 10 different areas of each sample using an ocular micrometer in a light microscope, and the MSTD and the thickness of germ cell layers were calculated for eight samples from each group.

\section{Detection of apoptosis}

Validated CIRP knockdown samples, negative controls and normal testes were prepared for the detection of apoptosis. Apoptosis was assessed by terminal deoxynucleotidyl transferase-mediated dUTP nick end labelling (TUNEL) using an in situ cell death detection kit and peroxidase (Roche, Mannheim, Germany). All samples were deparaffinized with xylene and alcohol. Sections were washed and microwaved for $5 \mathrm{~min}$ in the antigen retrieval solution $\left(0.1 \mathrm{~mol} \mathrm{l}^{-1}\right.$ pH 6.0 citrate buffers). Hydrogen peroxide (3\%) was employed to eliminate endogenous peroxidase activity. The TUNEL reaction mixture was applied to each slide so that the sections were completely covered, and the slides were incubated at $37{ }^{\circ} \mathrm{C}$ for $1 \mathrm{~h}$. Peroxidase (conjugated with horseradish peroxidase) was applied to the slides after blocking, and the samples were incubated at $37^{\circ} \mathrm{C}$ for $30 \mathrm{~min}$. A DAB kit was used as the staining agent. The stained slides were immersed in distilled water and kept in haematoxylin for 10-15 s. After dipping into xylene and alcohol, the slides were covered. The sections were evaluated using light microscopy. Seminiferous tubules containing three or more apoptotic cells by TUNEL staining were considered to be positive apoptotic tubules, and the apoptosis percentage was calculated by the ratio of positive seminiferous tubules to the total number of seminiferous tubules in a cross-section. ${ }^{26}$ Ten samples from each group were analysed.

\section{Western blotting}

We employed Western blotting to detect the mitogen-activated protein kinase (MAPK) pathway proteins in the siRNA group, negative control group and control group. Tissues were lysed in a tripledetergent RIPA buffer containing a protease inhibitor cocktail and sodium orthovanadate. Approximately $40 \mu \mathrm{g}$ of protein was separated by SDS-PAGE (10\%) and transferred to PVDF membranes. The membranes were blocked with $5 \%$ non-fat milk at room temperature for $1 \mathrm{~h}$ and incubated with anti-phospho-MAPK antibody (anti-phosphop44/42 was diluted 1:2000; the other antibodies were diluted $1: 1000$ in $5 \%$ bovine serum albumin) at $4{ }^{\circ} \mathrm{C}$ overnight. Following the secondary antibody incubation $(1: 2000)$ at room temperature for $45 \mathrm{~min}$, signals were visualized by enhanced chemiluminescence. The PVDF membranes were stripped, and then anti-MAPK $(1: 1000)$ and anti-GAPDH $(1: 1000)$ antibodies were used following the same processes as described above.

Table 1 siRNA sequences for CIRP

\begin{tabular}{lll}
\hline Name & Sense sequence & Antisense sequence \\
\hline SiRNA 1 & 5'-GAGACAGCUAUGACAGUUAUU-3' $^{\prime}$ & $5^{\prime}$-UAACUGUCAUAGCUGUCUCUU-3' \\
SiRNA 2 & 5'-GUGGUAAAGGACAGGGAGAUU-3' & $5^{\prime}$-UCUCCCUGUCCUUUACCACUU-3' \\
Negative control 1 & 5'-GCAUAAGUUGAUGACAGCAUU-3' & $5^{\prime}$-UGCUGUCAUCAACUUAUGCUU-3' \\
Negative control 2 & 5'-GAUAGAGAGAGAGGCGUGAUU-3' & $5^{\prime}$-UCACGCCUCUCUCUCUAUCUU-3' \\
\hline
\end{tabular}

Abbreviation: CIRP, cold-inducible RNA-binding protein. 


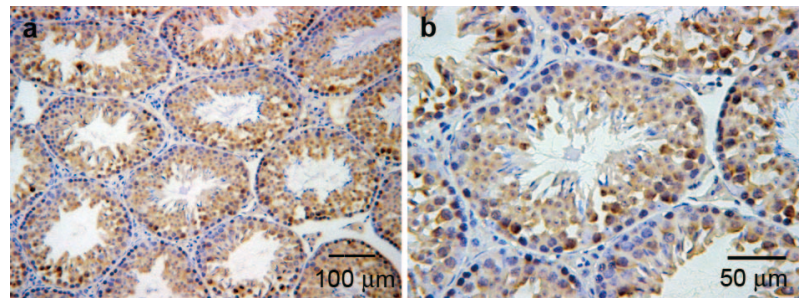

Figure 1 The expression profile of CIRP in testes. (a) CIRP was expressed in germ cells. (b) CIRP was mainly expressed in the primary spermatocytes, secondary spermatocytes and round spermatids but was not expressed in spermatogonia, elongating spermatids or Leydig cells. CIRP, cold-inducible RNAbinding protein.

\section{Statistical analysis}

Statistical analyses were performed using SPSS software 13.0 (SPSS corporation, Chicago, IL, USA), and all data were presented as the mean \pm standard deviation. One-way ANOVA was used as the test for statistical significance, and differences were considered significant if $P<0.05$.

\section{RESULTS}

The expression profile of CIRP in mouse testes

Immunohistochemical studies showed that CIRP was expressed in the testes under normal physiological conditions. CIRP was mainly expressed in the primary spermatocytes, secondary spermatocytes and round spermatids, but was not expressed in spermatogonia, elongating spermatids or Leydig cells. In the primary spermatocytes, CIRP was mainly located in the nucleus, but in round spermatids, it was present in the cytoplasm (Figure 1).

In vivo siRNA-mediated CIRP knockdown in the testes is a feasible and reliable research procedure

The infiltration efficiency test showed that the trypan blue appeared in almost all of the seminiferous tubules (Figure 2a). At a high magnification, trypan blue was detected in all the germ cell layers (Figure $\mathbf{2 b}$ and $2 \mathbf{c}$ ) and morphological changes were not observed.

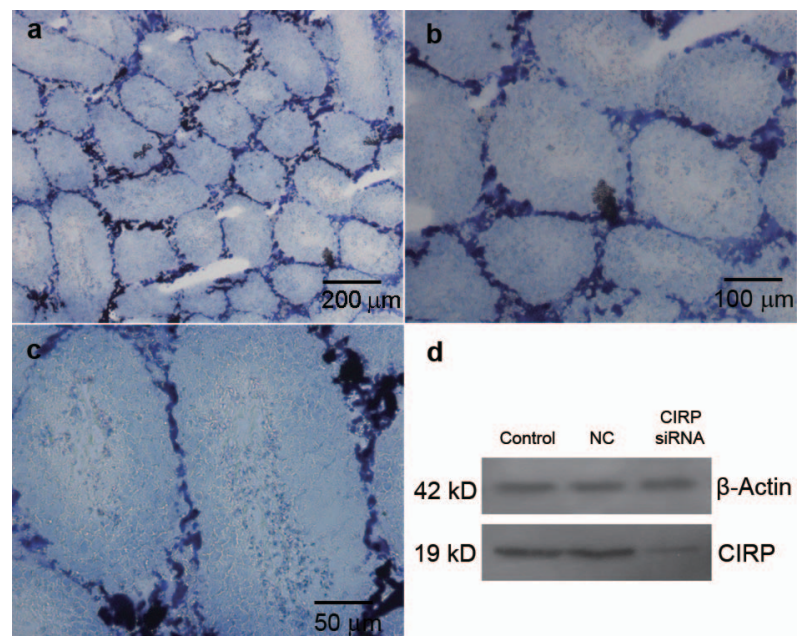

Figure 2 The knockdown validation of CIRP in testes. (a) Trypan blue appeared in almost all of seminiferous tubules in testes. (b) Trypan blue was detected in all germ cell layers. (c) At high magnification, trypan blue transfected into seminiferous tubules through microinjection did not result in morphologic changes. (d) Compared with control groups, CIRP was downregulated in CIRP siRNA group successfully. CIRP, cold-inducible RNA-binding protein; NC, negative control.
The tracer test suggested that microinjection was a feasible method for transfecting the testes with exogenous reagents.

In control group and the negative control group, the expression of CIRP was similar to each other. Compared with the control groups, CIRP was downregulated in the siRNA group, indicating that the siRNA sequences successfully interfered with the expression of CIRP (Figure 2d). We discovered that chemically modified siRNA delivered by Lipofectamine 2000 is a successful approach for knockdown of gene expression in the testes. We performed siRNA-mediated knockdown in vivo in samples from 12 testes, and 10 samples were confirmed to have downregulated CIRP. We performed the analyses described below in these samples.

\section{Downregulation of CIRP impairs spermatogenic function and} increases germ cell apoptosis

Both control groups showed normal testicular architecture and regular seminiferous tubular morphology with spermatogenesis, primary spermatocytes, secondary spermatocytes, spermatids and spermatozoa. TUNEL assays showed that most of the seminiferous tubules in the control groups contained less than three apoptotic cells (mainly spermatogonia) (Figure 3a-3f). The MSTD, the thickness of germ cell layers and the percentage of apoptotic seminiferous tubules in the control groups were not significantly different from each other.

Compared to the controls, there were marked decreases in the MSTD and the germ cell layer thickness in the siRNA group $(P<0.01)$ with severe distortion, extensive disorganisation, sloughing and a loss of maturation of germ cells in seminiferous tubules. The rate of apoptosis in the seminiferous tubules was higher than in the control groups $(P<0.01)$, and most of the apoptotic cells appeared in primary spermatocytes, secondary spermatocytes and round spermatids (Figure 3g-3i). The MSTD, the germ cell layer thickness and the percentage of apoptotic cells for each group are presented in Table 2.

\section{Downregulation of CIRP results in MAPK pathways activation in testes}

The above-described studies led us to investigate the possible mechanism of the apoptosis induced by downregulated CIRP. The $\mathrm{p} 44 / \mathrm{p} 42$, p38 and SAPK/JNK MAPK pathways were detected in both controls and the siRNA group. We found similar levels of both phosphorylated and total p44/42, p38 and SAPK/JNK in both control groups, suggesting that the microinjection had no effects on these pathways. Compared to the controls, the downregulation of CIRP increased the level of phospho-p44/42, phospho-p38 and phospho-SAPK/JNK in the siRNA group, but had no effects on total p44/p42 and SAPK/ JNK. The total amount of p38 protein was slightly increased in the siRNA group compared with the controls (Figure 4).

\section{DISCUSSION}

CIRP is a heat-sensitive protein that is expressed in the testes and is downregulated after heat stress caused by cryptorchidism, varicocele or environmental factors. It is well known that spermatogenic function is impaired after heat stress in the testes as a result of numerous pathological processes. In earlier studies, testes were subjected to heat stress to evaluate the functions of CIRP, ${ }^{18,19,27}$ but there is no direct evidence to indicate which pathological processes are caused by the downregulation of CIRP in heat-stressed testes. We employed the RNAi method to downregulate the expression of CIRP in the testes and found that CIRP was necessary for spermatogenesis. Downregulation of CIRP induced germ cell apoptosis, which was associated with the activation of the p44/p42, p38 and SAPK/JNK 


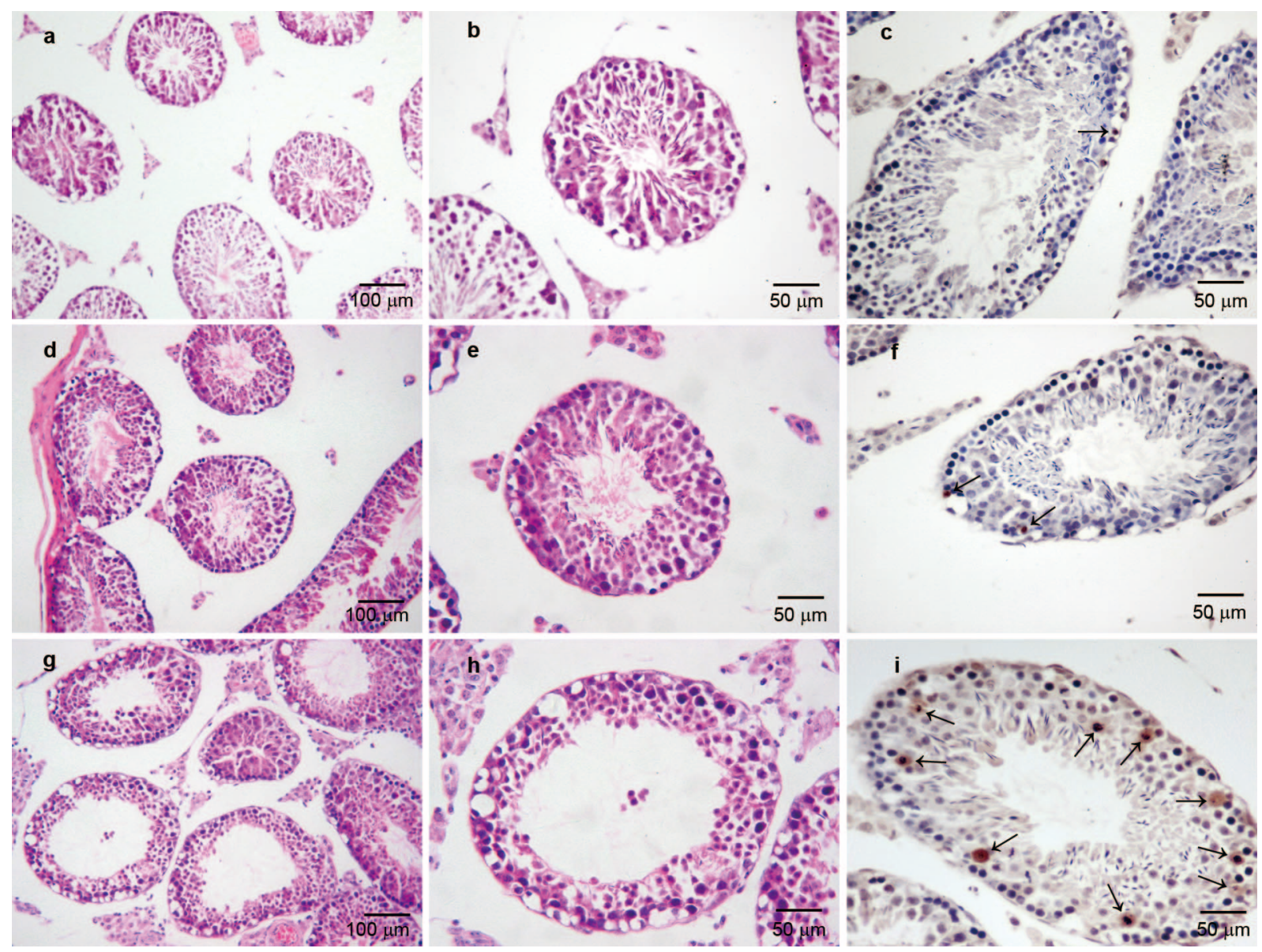

Figure 3 Downregulation of CIRP increased germ cell apoptosis in testes. Control group (a-c) and negative control group (d-f) showed the normal testicular architecture and morphology and most of the seminiferous tubules contained less than three apoptotic cells by TUNEL stain. In CIRP siRNA group (g-i), the testes showed severe distortion, extensive disorganization, sloughing and a loss of maturation of germ cells in seminiferous tubules. Apoptotic cells increased and most of them appeared in primary spermatocytes, secondary spermatocytes and round spermatids. The sections in TUNEL assays slightly tilted and the seminiferous tubules presented as elliptic profiles. Black arrows showed the apoptotic cells stained by TUNEL. CIRP, cold-inducible RNA-binding protein; TUNEL, terminal deoxynucleotidal transferase-mediated dUTP nick end labelling.

MAPK pathways. This model revealed that the downregulation of CIRP contributed to the germ cell death caused by heat stress.

We found that CIRP was expressed in primary spermatocytes, secondary spermatocytes and round spermatids, but not in spermatogonia, elongating spermatids or Leydig cells. These findings were in accord with Nishiyama's report in $1998 .{ }^{18}$ In primary spermatocytes, CIRP was primarily present in the nucleus but was cytoplasmic in round spermatids. This differential distribution of CIRP indicated that it may have different physiological functions in primary spermatocytes and round spermatids, ${ }^{28}$ the mechanisms of which are unclear. TUNEL staining showed that apoptosis occurred mainly in primary spermatocytes, secondary spermatocytes and round spermatids, which are the germ cells that express CIRP. Our results indicate that the downregulation of CIRP may induce germ cell apoptosis and contribute to the pathological processes in heat-stressed testes.

To investigate the possible mechanism of apoptosis caused by the downregulation of CIRP, we assessed the p44/p42, p38, SAPK/JNK MAPK pathways in the experimental groups. MAPK pathways are three of the major types of signalling pathways involved in the responses to heat stress, and p44/p42, p38 and SAPK/JNK have been shown to be activated after heat shock. ${ }^{29}$ The role of activated MAPKs in the testes differed from the roles reported for other cell lines. In various cell systems, $\mathrm{p} 44 / \mathrm{p} 42$ has been reported to be expressed in response to growth stimuli and to promote cell growth, and p38 and SAPK/JNK were shown to be activated in response to a variety of environmental stresses and inflammatory signals and to promote apoptosis and growth inhibition. ${ }^{30-32}$ In the testes, p44/p42 and SAPK/JNK were found to be activated in a testicular ischemia-reperfusion model, ${ }^{33,34}$ and their blockade resulted in less testicular damage. ${ }^{34}$ p44/p42 and p38 were found to be activated after testicular heat treatment, but inhibition of this activation had no discernible effect on heat-induced germ cell apoptosis, suggesting that it may be dispensable for heat-induced germ cell apoptosis. ${ }^{35,36}$ Furthermore, p38 mediates germ cell apoptosis after hormone deprivation. ${ }^{37}$ The possible roles of activated $\mathrm{p} 44 / \mathrm{p} 42$ in germ cell apoptosis are currently unclear and are controversial, but it is clear that activation of $\mathrm{p} 38$ and SAPK/JNK promotes apoptosis in the testes. p38 activates the $i N O S$ gene, ${ }^{38,39}$ resulting in increased NO production and subsequent activation of the cytochrome c-mediated death pathway. p38 and SAPK/JNK also induce apoptosis through the activation of the intrinsic mitochondria-dependent pathway. ${ }^{40}$ Activation of SAPK/JNK pathway has been correlated with the production of proinflammatory cytokines, ${ }^{33}$ such as tumour-necrosis factor, which affects not only the proinflammatory response, ${ }^{41}$ but also the immunoregulatory 
Table 2 MSTD, thickness of germ cell layers and apoptosis rate among three different groups

\begin{tabular}{lccc}
\hline Groups & MSTD $(\mu \mathrm{m}, \mathrm{n}=8)$ & Thickness of germ cell layers $(\mathrm{n}=8)$ & Apoptosis rate $(\%, \mathrm{n}=10)$ \\
\hline Control & $178.12 \pm 4.00$ & $8.65 \pm 0.29$ & $3.60 \pm 1.35$ \\
Negative control & $179.12 \pm 3.09$ & $8.50 \pm 0.18$ & $3.90 \pm 1.29$ \\
CIRP siRNA group & $156.50 \pm 1.51^{*}$ & $5.45 \pm 0.32 *$ & $28.30 \pm 5.21 *$ \\
\hline
\end{tabular}

Abbreviation: MSTD, mean seminiferous tubule diameter.

$* P<0.01$ vs. control groups (one-way ANOVA)
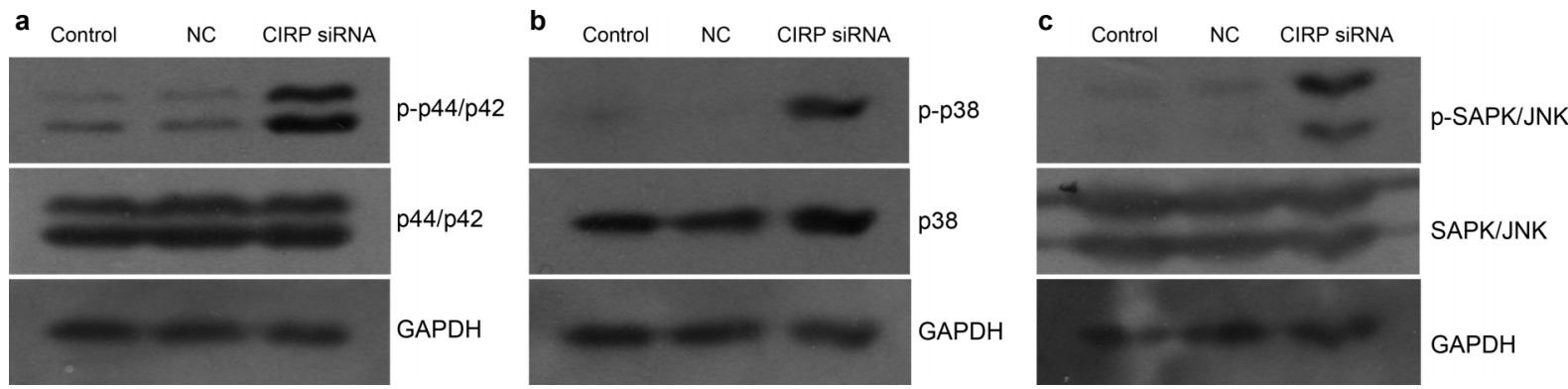

Figure 4 The expression of MAPKs in both controls and CIRP siRNA group. (a) p44/p42, (b) p38 and (c) SAPK/JNK MAPK pathways were detected in different groups. Phosphorylated and total p44/p42, p38 and SAPK/JNK were similar in control groups. In CIRP siRNA group, the level of phospho-p44/p42 (p-p44/p42), phospho-p38 ( $p-p 38$ ) and phospho-SAPK/JNK ( $p-S A P K / J N K$ ) were increased. Three independent experiments were performed and had similar results. CIRP, cold-inducible RNAbinding protein; MAPK, mitogen-activated protein kinase; NC, negative control.

response $^{42}$ and apoptosis. ${ }^{43}$ We discovered that p44/42, p38 and SAPK/JNK were activated in testes in which CIRP expression had been knocked down, similarly to the response to heat treatment, which demonstrates that the downregulation of CIRP may play an important role in the heat-stressed testes. Although we cannot determine whether this activation is direct or indirect, we can infer that the downregulation of CIRP may contribute to the activation of MAPK pathways in the heat-stressed testes and cause germ cell damage. The activation of MAPK pathways suggested that CIRP may be an upstream inhibitor of these pathways in the testes, but there is no direct evidence for this. The direct relationship between CIRP and the MAPK pathways in germ cells requires further study.

Microinjection was an important procedure in our study. We successfully performed microinjection of the testes to deliver the siRNA sequences. Microinjection has been used for stem cell transplantation and plasmid delivery, ${ }^{25,44-50}$ as described by Ogawa et al. ${ }^{25}$ in 1997. We found that siRNA delivered with the Lipofectamine 2000 reagent to the testes was an effective method for RNA interference research, and our recommended proportion of siRNA and Lipofectamine 2000 is $1: 3$ (m:v). The appropriate volume of siRNA complex was $30 \mu \mathrm{l}$ (for $\mathrm{BALB} / \mathrm{c}$ mice testes, about $100 \mathrm{mg}$ ), and the injection time should be $10 \mathrm{~min}$ or more.

In conclusion, CIRP plays an important role in spermatogenesis. The downregulation of CIRP may contribute to male infertility caused by heat stress possibly through the activation of MAPK pathways.

\section{AUTHOR CONTRIBUTIONS}

ZPX carried out the microinjection, siRNA in vivo studies and MAPK pathway analyses. XMZ conceived of the study, participated in its design and coordination and helped draft the manuscript. HZ participated in morphological studies and performed the statistical analysis. XJL participated in the TUNEL assay and drafted the manuscript. GYL carried out the immunohistochemical studies. XHW participated in the design of the study. All authors read and approved the final manuscript.

\section{COMPETING FINANCIAL INTERESTS}

The authors have no competing financial interests to declare.

\section{ACKNOWLEDGMENTS}

We thank Dr Yu Zeng and Dr Robert H Getzenberg (The Johns Hopkins University School of Medicine, USA) for providing the siRNA sequences of human CIRP. We thank all members of the research centre for helpful discussions and technical advice. This work was supported by the Fundamental Research Funds for the Central Universities (No. 201130302020013 to Zhi-Ping Xia) and Zhongnan Hospital of Wuhan University support (No. 63 to Xin-Min Zheng).

1 Tsujimura A, Fujita K, Komori K, Tanjapatkul P, Miyagawa $Y$ et al. Associations of homologous RNA-binding motif gene on the $X$ chromosome (RBMX) and its like sequence on chromosome 9 (RBMXL9) with non-obstructive azoospermia. Asian $\mathrm{J}$ Androl 2006; 8: 213-8.

2 Cho YS, Chennathukuzhi VM, Handel MA, Eppig J, Hecht NB. The relative levels of translin-associated factor X (TRAX) and testis brain RNA-binding protein determine their nucleocytoplasmic distribution in male germ cells. J Biol Chem 2004; 279: 31514-23.

3 Osman BA, Kawashima A, Tamba M, Satoh E, Kato Y et al. Localization of a novel RNAbinding protein, SKIV2L2, to the nucleus in the round spermatids of mice. J Reprod Dev 2011; 57: 457-67.

4 Yang Y, Xiao CY, A ZC, Zhang SZ, Li X et al. DAZ1/DAZ2 cluster deletion mediated by gr/gr recombination per se may not be sufficient for spermatogenesis impairment: a study of Chinese normozoospermic men. Asian J Androl 2006; 8: 183-7.

5 Burd CG, Dreyfuss G. Conserved structures and diversity of functions of RNA-binding proteins. Science 1994; 265: 615-21.

6 Bergeron D, Beauseigle D, Bellemare G. Sequence and expression of a gene encoding a protein with RNA-binding and glycine-rich domains in Brassica napus. Biochim Biophys Acta 1993; 1216: 123-5.

7 Wellmann S, Buhrer C, Moderegger E, Zelmer A, Kirschner R et al. Oxygen-regulated expression of the RNA-binding proteins RBM3 and CIRP by a HIF-1-independent mechanism. J Cell Sci 2004; 117: 1785-94.

8 Yoon SK, Hong JK, Choo SH, Song JY, Park HW et al. Adaptation of Chinese hamster ovary cells to low culture temperature: cell growth and recombinant protein production. J Biotechnol 2006; 122: 463-72.

9 Fornace AJ, Alamo IJ, Hollander MC. DNA damage-inducible transcripts in mammalian cells. Proc Nat/ Acad Sci USA 1988; 85: 8800-4.

10 Sheikh MS, Carrier F, Papathanasiou MA, Hollander MC, Zhan Q et al. Identification of several human homologs of hamster DNA damage-inducible transcripts. Cloning and characterization of a novel UV-inducible cDNA that codes for a putative RNA-binding protein. J Biol Chem 1997; 272: 26720-6. 
11 Yang C, Carrier F. The UV-inducible RNA-binding protein A18 (A18 hnRNP) plays a protective role in the genotoxic stress response. J Biol Chem 2001; 276: 47277-84.

12 Lleonart ME. A new generation of proto-oncogenes: cold-inducible RNA binding proteins. Biochim Biophys Acta 2010; 1805: 43-52.

13 Nishiyama $\mathrm{H}$, Itoh $\mathrm{K}$, Kaneko $\mathrm{Y}$, Kishishita M, Yoshida $\mathrm{O}$ et al. A glycine-rich RNA binding protein mediating cold-inducible suppression of mammalian cell growth. J Cell Biol 1997; 137: 899-908.

14 Saito K, Fukuda N, Matsumoto T, Iribe Y, Tsunemi A et al. Moderate low temperature preserves the stemness of neural stem cells and suppresses apoptosis of the cells via activation of the cold-inducible RNA binding protein. Brain Res 2010; 1358: 20-9.

15 Al-Fageeh MB, Smales CM. Control and regulation of the cellular responses to cold shock: the responses in yeast and mammalian systems. Biochem J 2006; 397: 247 59.

16 Fujita J. Cold shock response in mammalian cells. J Mol Microbiol Biotechnol 1999; 1: $243-55$.

17 Xue JH, Nonoguchi K, Fukumoto M, Sato T, Nishiyama $\mathrm{H}$ et al. Effects of ischemia and $\mathrm{H}_{2} \mathrm{O}_{2}$ on the cold stress protein CIRP expression in rat neuronal cells. Free Radic Biol Med 1999; 27: 1238-44.

18 Nishiyama H, Danno S, Kaneko Y, Itoh K, Yokoi H et al. Decreased expression of coldinducible RNA-binding protein (CIRP) in male germ cells at elevated temperature. $\mathrm{Am}$ J Pathol 1998; 152: 289-96.

19 Zhou KW, Zheng XM, Yang ZW, Zhang L, Chen HD. Overexpression of CIRP may reduce testicular damage induced by cryptorchidism. Clin Invest Med 2009; 32: E103-11.

20 lida T, Mine S, Fujimoto H, Suzuki K, Minami Y et al. Hypoxia-inducible factor-1alpha induces cell cycle arrest of endothelial cells. Genes Cells 2002; 7: 143-9.

21 Carmeliet P, Dor Y, Herbert JM, Fukumura D, Brusselmans K et al. Role of HIF-1alpha in hypoxia-mediated apoptosis, cell proliferation and tumour angiogenesis. Nature 1998; 394: 485-90.

22 Aitken RJ. Free radicals, lipid peroxidation and sperm function. Reprod Fertil Dev 1995; 7: 659-68.

23 Ong CN, Shen HM, Chia SE. Biomarkers for male reproductive health hazards: are they available? Toxicol Lett 2002; 134: 17-30.

24 Aitken RJ, Gordon E, Harkiss D, Twigg JP, Milne P et al. Relative impact of oxidative stress on the functional competence and genomic integrity of human spermatozoa. Biol Reprod 1998; 59: 1037-46.

25 Ogawa T, Arechaga JM, Avarbock MR, Brinster RL. Transplantation of testis germinal cells into mouse seminiferous tubules. Int J Dev Biol 1997; 41: 111-22.

26 Kanter M. Protective effects of melatonin on testicular torsion/detorsion-induced ischemia-reperfusion injury in rats. Exp Mol Pathol 2010; 89: 314-20.

27 Banks S, King SA, Irvine DS, Saunders PT. Impact of a mild scrotal heat stress on DNA integrity in murine spermatozoa. Reproduction 2005; 129: 505-14.

28 De Leeuw F, Zhang T, Wauquier C, Huez G, Kruys V et al. The cold-inducible RNAbinding protein migrates from the nucleus to cytoplasmic stress granules by a methylation-dependent mechanism and acts as a translational repressor. Exp Cell Res 2007; 313: 4130-44.

29 Nadeau SI, Landry J. Mechanisms of activation and regulation of the heat shocksensitive signaling pathways. Adv Exp Med Biol 2007; 594: 100-13.

30 Johnson GL, Lapadat R. Mitogen-activated protein kinase pathways mediated by ERK JNK, and p38 protein kinases. Science 2002; 298: 1911-2.

31 Cowan KJ, Storey KB. Mitogen-activated protein kinases: new signaling pathways functioning in cellular responses to environmental stress. J Exp Biol 2003; 206: $1107-15$
32 Wada T, Penninger JM. Mitogen-activated protein kinases in apoptosis regulation. Oncogene 2004; 23: 2838-49.

33 Lysiak JJ, Nguyen QA, Kirby JL, Turner TT. Ischemia-reperfusion of the murine testis stimulates the expression of proinflammatory cytokines and activation of c-jun $\mathrm{N}$-terminal kinase in a pathway to E-selectin expression. Biol Reprod 2003; 69: 202-10.

34 Minutoli L, Antonuccio P, Romeo C, Nicotina PA, Bitto A et al. Evidence for a role of mitogen-activated protein kinase $3 /$ mitogen-activated protein kinase in the development of testicular ischemia-reperfusion injury. Biol Reprod 2005; 73 730-6.

35 Jia Y, Castellanos J, Wang C, Sinha-Hikim I, Lue Y et al. Mitogen-activated protein kinase signaling in male germ cell apoptosis in the rat. Biol Reprod 2009; 80: 77180

36 Zhang XS, Zhang ZH, Guo SH, Yang W, Zhang ZQ et al. Activation of extracellular signal-related kinases 1 and 2 in Sertoli cells in experimentally cryptorchid rhesus monkeys. Asian J Androl 2006; 8: 265-72.

37 Vera Y, Erkkila K, Wang C, Nunez C, Kyttanen S et al. Involvement of p38 mitogenactivated protein kinase and inducible nitric oxide synthase in apoptotic signaling of murine and human male germ cells after hormone deprivation. Mol Endocrinol 2006; 20: 1597-609.

38 Bhat NR, Zhang $P$, Bhat AN. Cytokine induction of inducible nitric oxide synthase in an oligodendrocyte cell line: role of p38 mitogen-activated protein kinase activation. J Neurochem 1999; 72: 472-8.

39 Bhat NR, Feinstein DL, Shen Q, Bhat AN p38 MAPK-mediated transcriptional activation of inducible nitric-oxide synthase in glial cells. Roles of nuclear factors, nuclear factor kappa B, cAMP response element-binding protein, CCAAT/enhancerbinding protein-beta, and activating transcription factor-2. J Biol Chem 2002; 277: 29584-92.

40 Danial NN, Korsmeyer SJ. Cell death: critical control points. Cel/2004; 116: 205-19.

41 Fiers W. Tumor necrosis factor. Characterization at the molecular, cellular and in vivo level. FEBS Lett 1991; 285: 199-212.

42 Beutler B. TNF, immunity and inflammatory disease: lessons of the past decade. J Investig Med 1995; 43: 227-35.

43 Baker SJ, Reddy EP. Modulation of life and death by the TNF receptor superfamily. Oncogene 1998; 17: 3261-70.

$44 \mathrm{Lu} \mathrm{L}$, Lin M, Xu M, Zhou ZM, Sha JH. Gene functional research using polyethylenimine-mediated in vivo gene transfection into mouse spermatogenic cells. Asian J Androl 2006; 8: 53-9.

45 Yuan Z, Hou R, Wu J. Generation of mice by transplantation of an adult spermatogonial cell line after cryopreservation. Cell Prolif 2009; 42: 123-31.

46 Oatley JM, de Avila DM, McLean DJ, Griswold MD, Reeves JJ. Transplantation of bovine germinal cells into mouse testes. J Anim Sci 2002; 80: 1925-31.

47 Ogawa T, Dobrinski I, Avarbock MR, Brinster RL. Transplantation of male germ line stem cells restores fertility in infertile mice. Nat Med 2000; 6: 29-34.

48 Fujita K, Ohta H, Tsujimura A, Takao T, Miyagawa $Y$ et al. Transplantation of spermatogonial stem cells isolated from leukemic mice restores fertility without inducing leukemia. J Clin Invest 2005; 115: 1855-61.

49 McLean DJ, Johnston DS, Russell LD, Griswold MD. Germ cell transplantation and the study of testicular function. Trends Endocrinol Metab 2001; 12: 16-21.

50 Izadyar F, Creemers LB, van Dissel-Emiliani FM, van Pelt AM, de Rooij DG Spermatogonial stem cell transplantation. Mol Cell Endocrinol 2000; 169: 21-6. 